\title{
Constitutional implementation of social choice correspondences
}

Citation for published version (APA):

Peleg, B., Peters, H. J. M., \& Storcken, A. J. A. (2003). Constitutional implementation of social choice correspondences. METEOR, Maastricht University School of Business and Economics. METEOR Research Memorandum No. 025 https://doi.org/10.26481/umamet.2003025

Document status and date:

Published: 01/01/2003

DOI:

10.26481/umamet.2003025

Document Version:

Publisher's PDF, also known as Version of record

\section{Please check the document version of this publication:}

- A submitted manuscript is the version of the article upon submission and before peer-review. There can be important differences between the submitted version and the official published version of record.

People interested in the research are advised to contact the author for the final version of the publication, or visit the DOI to the publisher's website.

- The final author version and the galley proof are versions of the publication after peer review.

- The final published version features the final layout of the paper including the volume, issue and page numbers.

Link to publication

\footnotetext{
General rights rights.

- You may freely distribute the URL identifying the publication in the public portal. please follow below link for the End User Agreement:

www.umlib.nl/taverne-license

Take down policy

If you believe that this document breaches copyright please contact us at:

repository@maastrichtuniversity.nl

providing details and we will investigate your claim.
}

Copyright and moral rights for the publications made accessible in the public portal are retained by the authors and/or other copyright owners and it is a condition of accessing publications that users recognise and abide by the legal requirements associated with these

- Users may download and print one copy of any publication from the public portal for the purpose of private study or research.

- You may not further distribute the material or use it for any profit-making activity or commercial gain

If the publication is distributed under the terms of Article $25 \mathrm{fa}$ of the Dutch Copyright Act, indicated by the "Taverne" license above, 


\title{
Constitutional Implementation of Social Choice Correspondences
}

\author{
Bezalel Peleg* ${ }^{*}$ Hans Peters ${ }^{\dagger} \quad$ Ton Storcken ${ }^{\dagger}$
}

March 2003

\begin{abstract}
A game form constitutionally implements a social choice correspondence if it implements it in Nash equilibrium and, moreover, the associated effectivity functions coincide. This paper presents necessary and sufficient conditions for a unanimous social choice correspondence to be constitutionally implementable, and sufficient and almost necessary conditions for an arbitrary (but surjective) social choice correspondence to be constitutionally implementable. It is shown that the results apply to interesting classes of scoring and veto rules.
\end{abstract}

JEL classification numbers: 025,026

Keywords: Social choice correspondence, game form, effectivity function, constitutional implementation.

*Department of Mathematics \& Center for Rationality and Interactive Decision Theory, The Hebrew University of Jerusalem, Feldman Building, Givat-Ram, 91904 Jerusalem, Israel. E-mail: pelegba@math.huji.ac.il. Financial support from the Research School METEOR of the University of Maastricht, and from the Dutch Science Foundation NWO (grant no. B 46-476) is gratefully acknowledged.

${ }^{\dagger}$ Department of Quantitative Economics, University of Maastricht, P.O. Box 616, 6200 MD Maastricht, The Netherlands. E-mail: h.peters@ke.unimaas.nl, t.storcken@ke.unimaas.nl. 


\section{Introduction}

Starting point of this paper is the traditional social choice framework with a finite number of agents (or players) and a finite number of alternatives. A social choice correspondence assigns to each profile of preferences a nonempty set of alternatives. In a mechanism or game form, each player is endowed with a set of strategies, and an outcome function assigns to each profile of strategies an alternative. A game form implements a social choice correspondence (in Nash equilibrium) if for each profile of preferences the set of alternatives assigned by the social choice correspondence coincides with the set of Nash equilibrium outcomes of the associated game (cf. Hurwicz, 1972; Maskin, 1985; Danilov, 1992; Yamamoto, 1992).

The social choice correspondence can be seen as a centralized procedure that reflects an underlying constitution. More precisely, a social choice correspondence endows each group of agents with a certain 'constitutional' power. Formally, this power or constitution can be represented by the effectivity function derived from the social choice correspondence (cf. Gardenförs, 1981; Moulin and Peleg, 1982; Peleg, 1984; Peleg, 1998; Abdou and Keiding, 1991).

A game form is a decentralized procedure and also endows each group of agents (players) with a certain power, again represented by its associated effectivity function (cf. Gaertner et al., 1992). If a game form implements a given social choice correspondence, then the right alternatives - 'right' in terms of the constitution underlying the social choice correspondence - are attained in a decentralized manner (so by the agents' own choice) as long as a Nash equilibrium is played. If we do not want to rely on the players playing Nash equilibria, then we need additional restrictions on the game form that guarantee the maintenance of the constitution underlying the social choice correspondence.

Following Peleg and Winter (2002) we will formalize this by considering so-called constitutional implementation, which requires that the game form not only implements a social choice correspondence but also has the same associated effectivity function. In other words, the game form endows each group of agents with exactly the same power as the social choice correspondence.

The first main result is a tight characterization of all unanimous social choice correspondences that are constitutionally implementable. The conditions involved are (Maskin) monotonicity; full power of $n$-1-person coalitions 
( $n$ is the number of agents); and a condition requiring that if two disjoint coalitions are effective for two sets of alternatives, then every alternative must be in at least one of these sets. The second main result gives sufficient and 'almost' necessary conditions for constitutional implementation of social choice correspondences that are not necessarily unanimous but only surjective. By discussing exemplary classes of scoring rules and veto rules we show that this last result enables us to establish constitutional implementation of many interesting but non-unanimous rules.

The organization of the paper is as follows. After preliminaries in Section 2, we discuss constitutional implementation of unanimous and surjective social choice correspondences in Sections 3 and 4, respectively. In Section 5 we present the mentioned examples and show independence of the characterizing conditions. Section 6 concludes.

Notations. For a set $D, 2^{D}$ denotes the set of all subsets of $D$, and $P(D)$ the set of all nonempty subsets. By $|D|$ we denote the number of elements of $D$.

\section{Preliminaries}

Let $A$ be the nonempty (finite or infinite) set of alternatives. Throughout we assume $|A| \geq 2$, in order to avoid trivialities. A preference $R$ on $B \subseteq A$ is a complete, transitive, and antisymmetric binary relation on $B$. The set of all preferences on $B$ is denoted by $L(B)$. For $R \in L(A), R \mid B$ denotes the restriction to $B$. We will also use notations like $x R B$ (alternative $x$ is (weakly) preferred to all elements of $B$ ), $B^{\prime} R B$ (every alternative in $B^{\prime}$ is (weakly) preferred to every alternative in $B$ ), etc.

Let $N=\{1, \ldots, n\}$ be the (finite) set of players. Throughout we assume $n \geq 3$. An $n$-tuple $R^{N} \in L(A)^{N}$ is called a preference profile. Similarly, for a coalition $S \in P(N), R^{S}$ denotes a preference profile for $S$, i.e., an element of $L(A)^{S}$.

A social choice correspondence (SCC) $H$ assigns to every preference profile $R^{N}$ a nonempty subset $H\left(R^{N}\right)$ of $A$. An SCC $H$ is called surjective if for every $x \in A$ there is an $R^{N} \in L(A)^{N}$ such that $\{x\}=H\left(R^{N}\right)$. It is called unanimous if $H\left(R^{N}\right)=\{x\}$ for all $R^{N} \in L(A)^{N}$ and $x \in A$ such that $x R^{i} A$ for all $i \in N$. Obviously, unanimity of an SCC implies surjectivity. 
A game form is an $(n+1)$-tuple $\Gamma=\left(\Sigma^{1}, \ldots, \Sigma^{n}, \pi\right)$ where

(i) for each player $i \in N, \Sigma^{i}$ is a nonempty set of strategies;

(ii) $\pi: \Sigma^{1} \times \ldots \times \Sigma^{n} \rightarrow A$ is a surjective map called the outcome function.

By $\Sigma^{S}(S \in P(N))$ we denote the product $\prod_{i \in S} \Sigma^{i}$. An element of $\Sigma^{S}$ is called a strategy profile (for $S$ ). For $R^{N} \in L(A)^{N}$ the pair $\left(\Gamma, R^{N}\right)$ is a game in strategic form in the obvious way. A strategy profile $\sigma \in \Sigma^{N}$ is a Nash equilibrium of $\left(\Gamma, R^{N}\right)$ if $\pi(\sigma) R^{i} \pi\left(\sigma^{N \backslash\{i\}}, \tau^{i}\right)$ for all $\tau^{i} \in \Sigma^{i}$ and $i \in N$. The set of all Nash equilibria of the game $\left(\Gamma, R^{N}\right)$ is denoted by $\mathrm{NE}\left(\Gamma, R^{N}\right)$.

An effectivity function is a map $E: 2^{N} \rightarrow 2^{P(A)}$ such that

(i) $E(\emptyset)=\emptyset$;

(ii) $E(N)=P(A)$;

(iii) $A \in E(S)$ for every $S \in P(N)$.

An effectivity function $E$ is monotonic if $B \in E(S)$ implies $B^{\prime} \in E\left(S^{\prime}\right)$ for all $B, B^{\prime} \in P(A)$ and $S, S^{\prime} \in P(N)$ with $B \subseteq B^{\prime}$ and $S \subseteq S^{\prime}$. It is superadditive if $B \cap B^{\prime} \in E\left(S \cup S^{\prime}\right)$ for all $B, B^{\prime} \in P(A)$ and $S, S^{\prime} \in P(N)$ with $B \in E(S)$, $B^{\prime} \in E\left(S^{\prime}\right)$, and $S \cap S^{\prime}=\emptyset$.

Let $H$ be a surjective SCC. We associate with $H$ an effectivity function $E^{H}$, as follows. Define $E^{H}(\emptyset)=\emptyset$ and for $B \in P(A)$ and $S \in P(N)$ let $B \in$ $E^{H}(S)$ if there is a preference profile $R^{S} \in L(A)^{S}$ such that $H\left(R^{S}, Q^{N \backslash S}\right) \subseteq$ $B$ for every preference profile $Q^{N \backslash S} \in L(A)^{N \backslash S}$. Note that, in particular, $E^{H}(N)=P(A)$ by surjectivity of $H$, so $E^{H}$ is well defined. It is easy to see that $E^{H}$ is monotonic and superadditive.

Similarly, we can associate an effectivity function $E^{\Gamma}$ with a game form $\Gamma=\left(\Sigma^{1}, \ldots, \Sigma^{n}, \pi\right)$, as follows. Define $E^{\Gamma}(\emptyset)=\emptyset$ and for $B \in P(A)$ and $S \in P(N)$ let $B \in E^{\Gamma}(S)$ if there is a strategy profile $\sigma^{S} \in \Sigma^{S}$ such that $\pi\left(\sigma^{S}, \tau^{N \backslash S}\right) \in B$ for every strategy profile $\tau^{N \backslash S} \in \Sigma^{N \backslash S}$. In particular, $E^{\Gamma}(N)=P(A)$ by surjectivity of $\pi$, so $E^{\Gamma}$ is well defined. It is again easy to see that $E^{H}$ is monotonic and superadditive.

The game form $\Gamma$ implements the social choice correspondence $H$ (in Nash equilibrium) if $\pi\left(\mathrm{NE}\left(\Gamma, R^{N}\right)\right)=H\left(R^{N}\right)$ for every preference profile $R^{N} \in L(A)^{N}$. If $H$ is surjective, then $\Gamma$ constitutionally implements $H$ if $\Gamma$ implements $H$ and $E^{\Gamma}=E^{H}$. 
We conclude this section with introducing the following notation. For a preference $R \in L(A)$ and an alternative $a \in A$ let $L(a, R):=\{x \in A \mid a R x\}$ be the set of all alternatives to which $a$ is (weakly) preferred. Observe that $a \in L(a, R)$.

\section{Constitutional implementation of unanim- ous social choice correspondences}

The social choice correspondence $H$ is (Maskin) monotone if for all $R^{N}, Q^{N} \in$ $L(A)^{N}$ and $a \in H\left(R^{N}\right)$ such that $L\left(a, R^{i}\right) \subseteq L\left(a, Q^{i}\right)$ for all $i \in N$, we have $a \in H\left(Q^{N}\right)$. Monotonicity of $H$ is a necessary condition for implementability (cf. Maskin, 1985).

An alternative $a \in A$ is Pareto undominated in a preference profile $R^{N} \in$ $L(A)^{N}$ is there is no alternative $b \in A \backslash\{a\}$ with $b R^{i} a$ for all $i \in N$. The SCC $H$ is Pareto optimal if, for every $R^{N} \in L(A)^{N}, H\left(R^{N}\right)$ contains only Pareto undominated alternatives.

Lemma 3.1 Let the $S C C H$ be unanimous and monotone. Then $H$ is Pareto optimal.

Proof. Let $R^{N} \in L(A)^{N}$ and $a \in H\left(R^{N}\right)$ and suppose, contrary to what we wish to prove, that there is a $b \in A \backslash\{a\}$ such that $b R^{i} a$ for all $i \in N$. Consider the profile $\tilde{R}^{N}$ with $\tilde{R}^{i}\left|A \backslash\{b\}=R^{i}\right| A \backslash\{b\}$ and $b \tilde{R}^{i} A$ for all $i \in N$. By monotonicity, $a \in H\left(\tilde{R}^{N}\right)$, but by unanimity, $\{b\}=H\left(\tilde{R}^{N}\right)$. This is a contradiction, hence $a$ is Pareto undominated in $R^{N}$. We conclude that $H$ is Pareto optimal.

Call the SCC $H$ dictatorial if there is an $i \in N$, the dictator, such that, for all $R^{N} \in L(A)^{N}, H\left(R^{N}\right)=\{x\}$ where $x R^{i} A$. Obviously, a dictatorial social choice correspondence is unanimous and constitutionally implementable, e.g. by the game form in which every player announces a preference and the outcome function picks the best alternative of the dictator. The purpose of this section is to present necessary and sufficient conditions for a nondictatorial unanimous SCC to be constitutionally implementable.

Proposition 3.2 Let $H$ be an SCC. If $H$ is implementable, then $H$ is monotone. If $H$ is unanimous, nondictatorial, and constitutionally implementable then, moreover, the following two conditions hold. 
(U1) For all $B, B^{\prime} \in P(A)$ and $S, S^{\prime} \in P(N)$ with $B \in E^{H}(S), B^{\prime} \in E^{H}\left(S^{\prime}\right)$, and $S \cap S^{\prime}=\emptyset$, we have $B \cup B^{\prime}=A$.

(U2) For all $i \in N$, we have $E^{H}(N \backslash\{i\})=P(A)$.

Proof. For the first statement, see Maskin (1985). Condition (U2) follows from Theorem 6.2 in Peleg and Winter (2002). Condition (U1) follows from Lemma 3.1 above and Lemma 6.3 in Peleg and Winter (2002).

The interpretation of (U2) is obvious. Note that for anonymous SCCs (where the names of the players do not matter) condition (U1) is equivalent to the condition that $E^{H}(S) \neq\{A\}$ implies $|S|>n / 2$, for all $S \in P(N)$.

We will show that the conditions in Proposition 3.2 are also sufficient for constitutional implementation of a unanimous SCC. We start with an auxiliary result on monotone SCCs.

Lemma 3.3 Let $H$ be a monotone $S C C, S \in P(N)$, and $B \in E^{H}(S)$. Then there is a preference profile $\hat{R}^{S} \in L(A \backslash B)^{S}$ such that, if $R^{N} \in L(A)^{N}$ and for all $i \in S$ : (i) $R^{i} \mid A \backslash B=\hat{R}^{i}$; and (ii) $B R^{i} A \backslash B$, then $H\left(R^{N}\right) \subseteq B$.

Proof. Since $B \in E^{H}(S)$, there is a preference profile $\bar{R}^{S} \in L(A)^{S}$ such that $H\left(\bar{R}^{S}, R^{N \backslash S}\right) \subseteq B$ for all $R^{N \backslash S} \in L(A)^{N \backslash S}$. For every $i \in S$ define $\hat{R}^{i} \in L(A)$ by $\hat{R}^{i}\left|B=\bar{R}^{i}\right| B, \hat{R}^{i}\left|A \backslash B=\bar{R}^{i}\right| A \backslash B$, and $B \hat{R}^{i} A \backslash B$. Let $R^{N} \in L(A)^{N}$ be as in the statement of the lemma. Suppose $x \in H\left(R^{N}\right)$ for some $x \in A \backslash B$. Then by monotonicity, $x \in H\left(\bar{R}^{S}, R^{N \backslash S}\right)$, hence $x \in B$, a contradiction. Hence, $H\left(R^{N}\right) \subseteq B$.

We now present the main result of this section.

Proposition 3.4 Let $H$ be a unanimous and monotone social choice correspondence satisfying (U1) and (U2). Then $H$ is constitutionally implementable.

Proof. We construct a game form $\Gamma=\left(\Sigma^{1}, \ldots, \Sigma^{n}, \pi\right)$, as follows. For each $i \in N$, the strategy set $\Sigma^{i}$ consists of all five-tuples of the form $\left(R^{N}, x, t, S, B\right)$ where $R^{N} \in L(A)^{N}, x \in H\left(R^{N}\right), t \in\{0,1,2, \ldots\}, S \subseteq N$ with $i \in S$, and $B \in E^{H}(S)$. Let $\pi\left(\sigma^{N}\right) \in \Sigma^{N}$. For the definition of the outcome $\pi\left(\sigma^{N}\right)$ we distinguish three cases: 
( $\alpha$ ) There are $\bar{R}^{N} \in L(A)^{N}$ and $x \in H\left(\bar{R}^{N}\right)$ such that $\sigma^{i}=\left(\bar{R}^{N}, x, 0, N,\{x\}\right)$ for all $i \in N$. Then $\pi\left(\sigma^{N}\right):=x$.

( $\beta$ ) There are $\bar{R}^{N} \in L(A)^{N}, x \in H\left(\bar{R}^{N}\right)$, and $k \in N$ such that $\sigma^{i}=$ $\left(\bar{R}^{N}, x, 0, N,\{x\}\right)$ and $\sigma^{k}=\left(\cdot, x^{k}, \cdot, \cdot, \cdot\right) \neq \sigma^{i}$ for all $i \in N \backslash\{k\}$. Then $\pi\left(\sigma^{N}\right):=x^{k}$ if $x \bar{R}^{k} x^{k}$ and $\pi\left(\sigma^{N}\right):=x$ otherwise.

$(\gamma)$ Neither $(\alpha)$ nor $(\beta)$ hold. For the strategy profile $\sigma^{N}$, call a pair $(T, B)$ with $1<|T|<n-1,1 \leq|B|<|A|, T \in P(N)$, and $B \in E^{H}(T)$ decided if $\sigma^{t}=(\cdot, \cdot, 0, T, B)$ for all $t \in T$. Let $\mathcal{D}=\left\{\left(T^{1}, B^{1}\right), \ldots,\left(T^{\ell}, B^{\ell}\right)\right\}$ be the set of all decided pairs for $\sigma^{N}$. Let $D=\bigcap_{l=1}^{\ell} B^{l}$ if $\mathcal{D} \neq \emptyset$, and $D=A$ if $\mathcal{D}=\emptyset$. (Observe that, for $\bigcup_{l=1}^{\ell} T^{l} \neq \emptyset, D \in E^{H}\left(\bigcup_{l=1}^{\ell} T^{l}\right)$ by superadditivity of $E^{H}$. Otherwise, $D=A$.) Let $k$ be the player who announces the highest $t$ in $\sigma^{N}$; in case of a draw, take from those players the one with the highest number. If $\sigma^{k}=\left(R^{N}, \cdot, \cdot, \cdot, \cdot\right)$, then $\pi\left(\sigma^{N}\right):=x$ where $x \in D$ such that $x R^{k} D$.

We now show that $\Gamma$ constitutionally implements $H$.

(i) $\Gamma$ implements $H$.

Let $\tilde{R}^{N} \in L(A)^{N}$.

Take $a \in H\left(\tilde{R}^{N}\right)$. Consider the strategy $\sigma^{i}=\left(\tilde{R}^{N}, a, 0, N,\{a\}\right)$. By case $(\alpha)$, $\pi\left(\sigma^{N}\right)=a$. We show that $\sigma^{N}$ is a Nash equilibrium of $\left(\Gamma, \widetilde{R}^{N}\right)$. Suppose a player $k$ deviates to $\tau=\left(\cdot, x^{k}, \cdot, \cdot, \cdot\right)$. By case $(\beta), \pi\left(\sigma^{N \backslash\{k\}}, \tau\right)$ is equal to $a$ or equal to $x^{k}$ if $a \tilde{R}^{k} x^{k}$. Hence, $\pi\left(\sigma^{N}\right) \tilde{R}^{k} \pi\left(\sigma^{N \backslash\{k\}}, \tau\right)$. So $\sigma^{N} \in \operatorname{NE}\left(\Gamma, \tilde{R}^{N}\right)$, and $H\left(\tilde{R}^{N}\right) \subseteq \pi\left(\mathrm{NE}\left(\Gamma, \tilde{R}^{N}\right)\right)$.

For the converse, take $\sigma^{N} \in \mathrm{NE}\left(\Gamma, \tilde{R}^{N}\right)$ and let $a=\pi\left(\sigma^{N}\right)$. We distinguish three cases, according to the definition of $\pi\left(\sigma^{N}\right)$.

(a) There are $\bar{R}^{N} \in L(A)^{N}$ and $x \in H\left(\bar{R}^{N}\right)$ such that $\sigma^{i}=\left(\bar{R}^{N}, x, 0, N,\{x\}\right)$ for every $i \in N$.

Then $\pi\left(\sigma^{N}\right)=x=a$ and in particular $a \in H\left(\bar{R}^{N}\right)$. Take $k \in N$ and $y \in L\left(a, \bar{R}^{k}\right)$ arbitrary, and let $R^{N} \in L(A)^{N}$ be a preference profile with $y R^{j} A$ for all $j \in N$. By unanimity, $H\left(R^{N}\right)=\{y\}$, so $\tau=\left(R^{N}, y, 1, N,\{y\}\right)$ is a well defined strategy in $\Sigma^{k}$. By case $(\beta)$, we have $\pi\left(\sigma^{N \backslash\{k\}}, \tau\right)=y$, and since $\sigma^{N}$ is a Nash equilibrium in $\left(\Gamma, \tilde{R}^{N}\right)$, we have $\pi\left(\sigma^{N}\right) \tilde{R}^{k} \pi\left(\sigma^{N \backslash\{k\}}, \tau\right)$. Hence, $a \tilde{R}^{k} y$, and since $y$ was an arbitrary element of $L\left(a, \bar{R}^{k}\right)$, it follows 
that $L\left(a, \bar{R}^{k}\right) \subseteq L\left(a, \tilde{R}^{k}\right)$. Since also $k$ was arbitrary and $a \in H\left(\bar{R}^{N}\right)$, we have by monotonicity of $H$ that $a \in H\left(\tilde{R}^{N}\right)$.

(b) There are $\bar{R}^{N} \in L(A)^{N}, x \in H\left(\bar{R}^{N}\right)$, and $k \in N$, such that $\sigma^{i}=$ $\left(\bar{R}^{N}, x, 0, N,\{x\}\right)$ and $\sigma^{k} \neq \sigma^{i}$ for every $i \in N \backslash\{k\}$.

Let $\sigma^{k}=\left(\cdot, \cdot, t^{k}, \cdot, \cdot\right)$. Take an arbitrary $y \in A$ and a profile $R^{N}$ with $y R^{j} A$ for all $j \in N$. By unanimity, $H\left(R^{N}\right)=\{y\}$. For an arbitrary player $i \in N \backslash\{k\}$, consider the strategy $\tau^{i}=\left(R^{N}, y, t^{k}+1,\{i\}, A\right)$, so that, with $\tau^{i}$, player $i$ announces the largest number. Then $\pi\left(\sigma^{N \backslash\{i\}}, \tau^{i}\right)$ is determined by case $(\gamma)$. Since there are no decided pairs in this strategy profile, we have $D=A$ and $\pi\left(\sigma^{N \backslash\{i\}}, \tau^{i}\right)=y$. Since $\sigma^{N}$ is a Nash equilibrium of $\left(\Gamma, \tilde{R}^{N}\right)$, we have $\pi\left(\sigma^{N}\right) \tilde{R}^{i} \pi\left(\sigma^{N \backslash\{i\}}, \tau^{i}\right)$, so $\pi\left(\sigma^{N}\right) \tilde{R}^{i} y$. Since $y \in A$ and $i \in N \backslash\{k\}$ were arbitrary, we have

$$
\pi\left(\sigma^{N}\right) \tilde{R}^{j} A \text { for all } j \in N \backslash\{k\} .
$$

By condition (U2), $\{a\} \in E^{H}(N \backslash\{k\})$. Hence, by Lemma 3.3, there is a profile $\hat{R}^{N \backslash\{k\}} \in L(A \backslash\{a\})^{N \backslash\{k\}}$ such that $H\left(Q^{N \backslash\{k\}}, \tilde{R}^{k}\right)=\{a\}$, where $a Q^{i} A$ and $Q^{i} \mid A \backslash\{a\}=\hat{R}^{i}$ for all $i \in N \backslash\{k\}$. By (1) and monotonicity, $a=\pi\left(\sigma^{N}\right) \in H\left(\tilde{R}^{N}\right)$.

(c) Neither $(\alpha)$ nor $(\beta)$.

Let $\mathcal{D}=\left\{\left(T^{1}, B^{1}\right), \ldots,\left(T^{\ell}, B^{\ell}\right)\right\}, D$, and $k$ be as in the definition of case $(\gamma)$. Let $\tau^{i}=\left(R^{N}, y, t^{k}+1,\{i\}, A\right)$ for an arbitrary $i \in N$ be defined exactly as in case (b) above.

If $\mathcal{D}=\emptyset$ then $D=A$. Since $\pi\left(\sigma^{N}\right) \tilde{R}^{i} \pi\left(\sigma^{N \backslash\{i\}}, \tau^{i}\right)$ and $\pi\left(\sigma^{N \backslash\{i\}}, \tau^{i}\right)=y$, we have $\pi\left(\sigma^{N}\right) \tilde{R}^{i} y$. Since both $i \in N$ and $y \in A$ were arbitrary, we have $\pi\left(\sigma^{N}\right) \tilde{R}^{i} A$ for all $i \in N$. By unanimity, $a=\pi\left(\sigma^{N}\right) \in H\left(\tilde{R}^{N}\right)$.

If $\mathcal{D} \neq \emptyset$ then let, for each $h \in\{1, \ldots, \ell\}, D^{h}:=\bigcap\left\{B^{l} \mid l \in\{1, \ldots, \ell\} \backslash\{h\}\right\}$, with $D^{1}:=A$ if $\ell=1$. Consider $h \in\{1, \ldots, \ell\}$ and $i \in T^{h}$. Take $y$ (as in $\left.\tau^{i}\right)$ in $D^{h}$. Then $\pi\left(\sigma^{N \backslash\{i\}}, \tau^{i}\right)=y$ by case $(\gamma)$. As $\pi\left(\sigma^{N}\right) \tilde{R}^{i} \pi\left(\sigma^{N \backslash\{i\}}, \tau^{i}\right)$, and $y \in D^{h}, h$, and $i \in T^{h}$ were arbitrary, we have:

$$
\pi\left(\sigma^{N}\right) \tilde{R}^{i} D^{h} \text { for every } h \in\{1, \ldots, \ell\} \text { and } i \in T^{h} .
$$

In exactly the same way we prove:

$$
\pi\left(\sigma^{N}\right) \tilde{R}^{i} D \text { for all } i \in N \backslash \bigcup_{l=1}^{\ell} T^{l} .
$$


By Lemma 3.3, for every $h \in\{1, \ldots, \ell\}$ there is an $\hat{R}^{T^{h}} \in L\left(A \backslash B^{h}\right)^{T^{h}}$ such that, for all $Q^{N \backslash T^{h}} \in L(A)^{N \backslash T^{h}}$, we have

$$
H\left(\dot{R}^{T^{h}}, Q^{N \backslash T^{h}}\right) \subseteq B^{h},
$$

where $\left(B^{h} \backslash D^{h}\right) \dot{R}^{i} \pi\left(\sigma^{N}\right) \dot{R}^{i}\left(D \backslash\left\{\pi\left(\sigma^{N}\right)\right\}\right) \dot{R}^{i}\left(D^{h} \backslash B^{h}\right)$ and $\dot{R}^{i} \mid A \backslash B^{h}=\hat{R}^{i}$, for every $h \in\{1, \ldots, \ell\}$ and $i \in T^{h}$. By superadditivity it follows, in particular, that

$$
H\left(\dot{R}^{T^{1}}, \ldots, \dot{R}^{T^{\ell}}, \tilde{R}^{N \backslash \bigcup_{h=1}^{\ell} T^{h}}\right) \subseteq D .
$$

Since, by construction, $\pi\left(\sigma^{N}\right) \dot{R}^{i} D$ for all $i \in \bigcup_{h=1}^{\ell} T^{h}$ and, by $(3), \pi\left(\sigma^{N}\right) \tilde{R}^{i} D$ for all $i \in N \backslash \bigcup_{h=1}^{\ell} T^{h}$, we have by Pareto optimality (Lemma 3.1) that

$$
H\left(\dot{R}^{T^{1}}, \ldots, \dot{R}^{T^{\ell}}, \tilde{R}^{N \backslash \bigcup_{h=1}^{\ell} T^{h}}\right)=\left\{\pi\left(\sigma^{N}\right)\right\}=\{a\} .
$$

Let $h \in\{1, \ldots, \ell\}$ and $i \in T^{h}$. By (U1), $B^{h} \cup D^{h}=A$, hence $L\left(a, \dot{R}^{i}\right)=$ $A \backslash\left(B^{h} \backslash D^{h}\right)=D^{h}$. Since, by $(2), D^{h} \subseteq L\left(a, \tilde{R}^{i}\right)$, we have $L\left(a, \dot{R}^{i}\right) \subseteq$ $L\left(a, \tilde{R}^{i}\right)$. Since $h$ and $i \in T^{h}$ were arbitrary, (4) and monotonicity imply $a \in H\left(\tilde{R}^{N}\right)$.

By cases (a), (b), and (c), we have $\pi\left(\operatorname{NE}\left(\Gamma, \tilde{R}^{N}\right)\right) \subseteq H\left(\tilde{R}^{N}\right)$. This completes the proof that $\Gamma$ implements $H$.

(ii) $E^{\Gamma}=E^{H}$.

First, let $k \in N$ and consider $N \backslash\{k\}$. By (U2) it is sufficient to prove $E^{\Gamma}(N \backslash\{k\})=P(A)$. Let $x \in A$ arbitrary. By monotonicity of $E^{\Gamma}$ it is sufficient to prove that $E^{\Gamma}(N \backslash\{k\})$ contains $\{x\}$. By (U2) we can take a preference profile $\bar{R}^{N} \in L(A)^{N}$ such that $H\left(\bar{R}^{N}\right)=\{x\}$ and $A \bar{R}^{k} x$. For every $i \in N \backslash\{k\}$ let $\sigma^{i}=\left(\bar{R}^{N}, x, 0, N,\{x\}\right)$. By cases $(\alpha)$ and $(\beta)$ we have $\pi\left(\sigma^{N \backslash\{k\}}, \tau^{k}\right)=x$ for all $\tau^{k} \in \Sigma^{k}$. Hence, $E^{\Gamma}(N \backslash\{k\})=\{x\}$, as was to be proved.

Second, since we have just proved $E^{\Gamma}(N \backslash\{k\})=E^{H}(N \backslash\{k\})=P(A)$ for all $k \in N$, it follows by superadditivity of $E^{\Gamma}$ and $E^{H}$ that $E^{\Gamma}(\{k\})=$ $E^{H}(\{k\})=\{A\}$ for every player $k$.

It remains to consider coalitions $S$ with $1<|S|<n-1$. Let $B \in$ $E^{H}(S)$, without loss of generality $B \neq A$. Consider an $x \in A$ and a strategy profile $\bar{R}^{N}$ with $x \bar{R}^{i} A$ for all $i \in N$, so $H\left(\bar{R}^{N}\right)=\{x\}$ by unanimity. Let $\sigma^{i}=\left(\bar{R}^{N}, x, 0, S, B\right)$ for every $i \in S$. Since $1<|S|<n-1, \pi\left(\sigma^{S}, \tau^{N \backslash S}\right)$ is determined by case $(\gamma)$ for every $\tau^{N \backslash S} \in \Sigma^{N \backslash S}$. Obviously, $(S, B)$ is a decided 
pair in every such strategy profile $\left(\sigma^{S}, \tau^{N \backslash S}\right)$, so that $\pi\left(\sigma^{S}, \tau^{N \backslash S}\right) \in B$. This proves $B \in E^{\Gamma}(S)$.

Finally, let $B \in E^{\Gamma}(S)$, without loss of generality $B \neq A$. Take a strategy profile $\sigma^{S} \in \Sigma^{S}$ with $\pi\left(\sigma^{S}, \tau^{N \backslash S}\right) \in B$ for all $\tau^{N \backslash S} \in \Sigma^{N \backslash S}$. With $\bar{R}^{N}$ and $x$ as in the preceding paragraph, consider a strategy $\tau^{i}=\left(\bar{R}^{N}, x, t^{i},\{i\}, A\right) \in \Sigma^{i}$ for every $i \in N \backslash S$. Since $|S|<n-1, \pi\left(\sigma^{S}, \tau^{N \backslash S}\right)$ is determined by case $(\gamma)$. Let $\left(T^{1}, B^{1}\right), \ldots,\left(T^{\ell}, B^{\ell}\right), D$, and $k$ be as in the definition of $\pi\left(\sigma^{S}, \tau^{N \backslash S}\right)$ in $(\gamma)$. By the choice of $\tau^{i}$ for $i \in N \backslash S$, we have $\bigcup_{l=1}^{\ell} T^{l} \subseteq S$. By varying $x$ in the definition of $\tau^{i}$ over $D$ and choosing $t^{i}$ high enough, we obtain that the range of $\pi\left(\sigma^{S}, \cdot\right)$ is $D$. Hence, $D \subseteq B$. By superadditivity, $D=\bigcap_{l=1}^{\ell} B^{l} \in$ $E^{H}\left(\bigcup_{l=1}^{\ell} T^{l}\right)$. Thus, by monotonicity of $E^{H}, B \in E^{H}(S)$.

This completes the proof of (ii), and of the proposition.

By combining Propositions 3.2 and 3.4 the following corollary results.

Corollary 3.5 Let $H$ be a unanimous and nondictatorial social choice correspondence. Then $H$ is constitutionally implementable if and only if it is monotone and satisfies (U1) and (U2).

\section{Constitutional implementation of surject- ive social choice correspondences}

In this section we consider the larger class of surjective social choice correspondences. As observed, surjectivity is implied by unanimity. In the context of implementability and, thus, of (Maskin) monotonicity, surjectivity implies the following weakening of unanimity. Call the SCC $H$ weakly unanimous if $x \in H\left(R^{N}\right)$ for all $R^{N} \in L(A)^{N}$ and $x \in A$ such that $x R^{i} A$ for all $i \in N$.

Lemma 4.1 Let the $S C C H$ be monotone and surjective. Then $H$ is weakly unanimous.

Proof. Let $x \in A$ and $R^{N} \in L(A)^{N}$ with $x R^{i} A$ for all $i \in N$. By surjectivity there is a profile $\hat{R}^{N} \in L(A)^{N}$ with $\{x\}=H\left(\hat{R}^{N}\right)$. By monotonicity, $x \in$ $H\left(R^{N}\right)$.

In this section we give a set of necessary and a set of sufficient conditions for constitutional implementation of a surjective social choice correspondence. We start with a necessary condition. 
Lemma 4.2 Let the SCCH be surjective and constitutionally implementable.

Then

(S1) for all $i \in N, R^{N} \in L(A)^{N}$, and $a \in H\left(R^{N}\right)$, we have $L\left(a, R^{i}\right) \in$ $E^{H}(N \backslash\{i\})$.

Proof. See Peleg and Winter (2002, Lemma 3.4).

We will show (Proposition 4.3 below) that by adding the following condition we obtain a set of sufficient conditions for constitutional implementation of a surjective $\mathrm{SCC} H$.

(S2) Let $T^{1}, \ldots, T^{\ell}$ be pairwise disjoint coalitions $(\ell \geq 1), B^{l} \in E^{H}\left(T^{l}\right)$ for each $l=1, \ldots, \ell, x \in D:=\bigcap_{l=1}^{\ell} B^{l}$, and $R^{N} \in L(A)^{N}$. For each $h \in\{1, \ldots, \ell\}$ define $D^{h}:=\bigcap_{l \in\{1, \ldots, \ell\} \backslash\{h\}} B^{l}$, and $D^{1}:=A$ if $\ell=1$. Suppose that $x R^{i} D^{h}$ for all $i \in T^{h}$ and $h \in\{1, \ldots, \ell\}$, and $x R^{i} D$ for all $i \in N \backslash \bigcup_{l=1}^{\ell} T^{l}$. Then $x \in H\left(R^{N}\right)$.

Proposition 4.3 Let $H$ be a monotone and surjective SCC satisfying (S1) and (S2). Then $H$ is constitutionally implementable.

Proof. We use a game form $\Gamma$ that is almost identical to the one in the proof of Proposition 3.4. There are two differences.

First, in case $(\beta)$, let $\sigma^{k}=\left(\cdot, x^{k}, \cdot, S, B\right)$ be the strategy of the 'deviating' player. If $S \neq\{k\}$, then $\pi\left(\sigma^{N}\right)$ is as before, i.e., $\pi\left(\sigma^{N}\right):=x^{k}$ if $x^{k} \in L\left(x, \bar{R}^{k}\right)$ and $\pi\left(\sigma^{N}\right):=x$ otherwise. If $S=\{k\}$ (and, thus, $B \in E^{H}(\{k\})$ ), then by (S1) and superadditivity of $E^{H}, L\left(x, \bar{R}^{k}\right) \cap B \neq \emptyset$; let $z \in L\left(x, \bar{R}^{k}\right) \cap B$ with $z \bar{R}^{k}\left(L\left(x, \bar{R}^{k}\right) \cap B\right)$. Now define $\pi\left(\sigma^{N}\right):=x^{k}$ if $x^{k} \in L\left(x, \bar{R}^{k}\right) \cap B, \pi\left(\sigma^{N}\right):=x$ if $x^{k} \notin L\left(x, \bar{R}^{k}\right) \cap B$ and $x \in B$, and $\pi\left(\sigma^{N}\right):=z$ if $x^{k} \notin L\left(x, \bar{R}^{k}\right) \cap B$ and $x \notin B$.

Second, in case $(\gamma)$ we allow $|T|=1$ or $|T|=n-1$ for a decided pair $(T, B)$.

We first show:

$\Gamma$ implements $H$.

Let $\tilde{R}^{N} \in L(A)^{N}$. The proof that $H\left(\tilde{R}^{N}\right) \subseteq \pi\left(\mathrm{NE}\left(\Gamma, \tilde{R}^{N}\right)\right)$ is almost identical to the corresponding part in the proof of Proposition 3.4. For the converse, take $\sigma^{N} \in \mathrm{NE}\left(\Gamma, \tilde{R}^{N}\right)$ and let $a=\pi\left(\sigma^{N}\right)$. We distinguish three cases, according to the definition of $\pi\left(\sigma^{N}\right)$. 
(a) There are $\bar{R}^{N} \in L(A)^{N}$ and $x \in H\left(\bar{R}^{N}\right)$ such that $\sigma^{i}=\left(\bar{R}^{N}, x, 0, N,\{x\}\right)$ for every $i \in N$.

Then $\pi\left(\sigma^{N}\right)=x=a$ and in particular $a \in H\left(\bar{R}^{N}\right)$. Take $k \in N$ and $y \in L\left(a, \bar{R}^{k}\right)$ arbitrary, and let $R^{N} \in L(A)^{N}$ be a preference profile with $y R^{j} A$ for all $j \in N$. By weak unanimity (Lemma 4.1), $y \in H\left(R^{N}\right)$, so $\tau=\left(R^{N}, y, 1, N,\{y\}\right)$ is a well defined strategy in $\Sigma^{k}$. By case $(\beta)$, we have $\pi\left(\sigma^{N \backslash\{k\}}, \tau\right)=y$, and since $\sigma^{N}$ is a Nash equilibrium in $\left(\Gamma, \tilde{R}^{N}\right)$, we have $\pi\left(\sigma^{N}\right) \tilde{R}^{k} \pi\left(\sigma^{N \backslash\{k\}}, \tau\right)$. Hence, $a \tilde{R}^{k} y$, and since $y$ was an arbitrary element of $L\left(a, \bar{R}^{k}\right)$, it follows that $L\left(a, \bar{R}^{k}\right) \subseteq L\left(a, \tilde{R}^{k}\right)$. Since also $k$ was arbitrary and $a \in H\left(\bar{R}^{N}\right)$, we have by monotonicity of $H$ that $a \in H\left(\tilde{R}^{N}\right)$.

(b) There are $\bar{R}^{N} \in L(A)^{N}, x \in H\left(\bar{R}^{N}\right)$, and $k \in N$, such that $\sigma^{i}=$ $\left(\bar{R}^{N}, x, 0, N,\{x\}\right)$ and $\sigma^{k} \neq \sigma^{i}$ for every $i \in N \backslash\{k\}$.

First consider player $k$. Just as in part (a), we derive that $a \tilde{R}^{k} L\left(x, \bar{R}^{k}\right)$.

Next, consider an arbitrary player $i \in N \backslash\{k\}$. Let player $k$ announce $t^{k}$ in $\sigma^{k}$ and consider a strategy $\tau^{i} \in \Sigma^{i}$ of the form $\tau^{i}=\left(\tilde{R}^{N}, \cdot, t^{k}+1,\{i\}, A\right)$. Then $\pi\left(\sigma^{N \backslash\{i\}}, \tau^{i}\right)$ is determined by case $(\gamma)$. In particular, $\pi\left(\sigma^{N \backslash\{i\}}, \tau^{i}\right) \tilde{R}^{i} B$, where the set $B$ is equal to $A$ if there is no decided pair, or given by $B \in$ $E^{H}(\{k\})$ if $\sigma^{k}$ is of the form $\sigma^{k}=(\cdot, \cdot, 0,\{k\}, B)$ (in which case $(\{k\}, B)$ is the only decided pair). Then $a \in B$ and, since $\sigma^{N}$ is a Nash equilibrium, $a \tilde{R}^{i} B$.

Now consider the pairs $\left(N \backslash\{k\}, L\left(x, \bar{R}^{k}\right)\right)$ and $(\{k\}, B)$. Then $B \in$ $E^{H}(\{k\})$, and, as derived, $a \tilde{R}^{i} B$ for all $i \in N \backslash\{k\}$. By (S1), $L\left(x, \bar{R}^{k}\right) \in$ $E^{H}(N \backslash\{k\})$. Since $a=\pi\left(\sigma^{N}\right)$ is equal to $x$ or an element of $L\left(x, \bar{R}^{k}\right)$, we have $a \in L\left(x, \bar{R}^{k}\right)$. Moreover, as derived, $a \tilde{R}^{k} L\left(x, \bar{R}^{k}\right)$. By (S2), it follows that $a \in H\left(\tilde{R}^{N}\right)$.

(c) Neither $(\alpha)$ nor $(\beta)$.

Let $\mathcal{D}=\left\{\left(T^{1}, B^{1}\right), \ldots,\left(T^{\ell}, B^{\ell}\right)\right\}, D$, and $k$ be as in the definition of case $(\gamma)$. Let $\tau^{i} \in \Sigma^{i}$ be of the form $\tau^{i}=\left(\cdot, \cdot, t^{k}+1,\{i\}, A\right)$ for an arbitrary $i \in N$. By considering deviations $\tau^{i}$ from the Nash equilibrium $\sigma^{N}$ for a player $i \in T^{l}$ for some $l \in\{1, \ldots, \ell\}$, we obtain $\pi\left(\sigma^{N}\right) \tilde{R}^{i} D^{l}$. By considering deviations $\tau^{i}$ for a player $i \in N \backslash \bigcup_{l=1}^{\ell} T^{l}$, we obtain $\pi\left(\sigma^{N}\right) \tilde{R}^{i} D$. By (S2), $a=\pi\left(\sigma^{N}\right) \in H\left(\tilde{R}^{N}\right)$.

(ii) $E^{\Gamma}=E^{H}$.

For $S=N, E^{\Gamma}(N)=E^{H}(N)=P(A)$ by definition.

Let $|S|=1$, say $S=\{k\}$. 
Let $B \in E^{H}(\{k\})$ and consider a strategy $\sigma^{k}$ of the form $\sigma^{k}=(\cdot, \cdot, 0,\{k\}, B) \in \Sigma^{k}$. Then, by case $(\beta)$ or case $(\gamma), \pi\left(\sigma^{k}, \tau^{N \backslash\{k\}}\right) \in B$ for all $\tau^{N \backslash\{k\}} \in \Sigma^{N \backslash\{k\}}$. Hence, $B \in E^{\Gamma}(\{k\})$.

Next, assume $B \in E^{\Gamma}(\{k\})$. Then there is a strategy $\sigma^{k} \in \Sigma^{k}$ such that $\pi\left(\sigma^{k}, \tau^{N \backslash\{k\}}\right) \in B$ for all $\tau^{N \backslash\{k\}} \in \Sigma^{N \backslash\{k\}}$. Suppose $\sigma^{k}=\left(\cdot, \cdot, t^{k}, \cdot, \cdot\right)$. By considering, for all $i \in N \backslash\{k\}$, a strategy $\tau^{i} \in \Sigma^{i}$ of the form $\tau^{i}=$ $\left(\bar{R}^{N}, x, t^{k}+1, N \backslash\{k\}, A\right)$ it follows by case $(\gamma)$ that there must be a set $B^{\prime} \subseteq B$ with $B^{\prime} \in E^{H}(\{k\})$ such that $\sigma^{k}$ has the form $\sigma^{k}=\left(\cdot, \cdot, 0,\{k\}, B^{\prime}\right)$. By monotonicity, $B \in E^{H}(\{k\})$.

Let $1<|S| \leq n-1$.

For $B \in E^{H}(S)$, consider a strategy profile $\sigma^{S}$ for $S$ with each $\sigma^{i}$ of the form $\sigma^{i}=(\cdot, \cdot, 0, S, B)$. By case $(\gamma)$, we obtain $B \in E^{\Gamma}(S)$.

Next, assume $B \in E^{\Gamma}(S)$. If $|S|<n-1$, then there must be $T^{1}, \ldots, T^{\ell}$, $B^{1}, \ldots, B^{\ell}$, and $D$ as in case $(\gamma)$, with $D \subseteq B$ and $\bigcup_{l=1}^{\ell} T^{l} \subseteq S$. By superadditivity and monotonicity, $B \in E^{H}(S)$. Finally, suppose $|S|=n-1$, say $S=N \backslash\{k\}$. Then there is a strategy profile $\sigma^{S}$ such that $\pi\left(\sigma^{S}, \tau^{k}\right) \in B$ for all $\tau^{k} \in \Sigma^{k}$. Consider a strategy $\tau^{k} \in \Sigma^{k}$ of the form $\tau^{k}=(\cdot, \cdot, \cdot,\{k\}, A)$. If $\pi\left(\sigma^{S}, \tau^{k}\right)$ is determined by case $(\gamma)$, then, as before and with notations as in $(\gamma), D \subseteq B$ (by varying $\tau^{k}$ ) and $\bigcup_{l=1}^{\ell} T^{l} \subseteq S$, so by superadditivity and monotonicity, $B \in E^{H}(S)$. If $\pi\left(\sigma^{S}, \tau^{k}\right)$ is determined by case $(\beta)$, then each $\sigma^{i}$ is of the form $\sigma^{i}=\left(\bar{R}^{N}, x, 0, N,\{x\}\right)$, and by varying $\tau^{k}$ we obtain $L\left(x, \bar{R}^{k}\right) \subseteq B$. Hence, $B \in E^{H}(S)$ by monotonicity and (S1).

This completes the proof of this case and of the proposition.

We know that the conditions in Proposition 4.3, apart from (S2), are also necessary for constitutional implementation of a social choice correspondence. Concerning (S2), we can show that its conclusion is necessary if the point $x$ in the set $D$ can be 'reached', e.g., if the set $D$ is 'minimal'. For an effectivity function $E$, a coalition $S$ and a set $B \in E(S)$, call $B$ minimal if no proper subset of $B$ is in $E(S)$.

Lemma 4.4 Let $H$ be a surjective $S C C$, let $\Gamma$ be a constitutional implementation of $H$, and let the premise of (S2) be fulfilled with $D$ minimal in $E^{H}\left(\bigcup_{l=1}^{\ell} T^{l}\right)$. Then $x \in H\left(R^{N}\right)$.

Proof. Since $\Gamma$ constitutionally implements $H$, there are $\sigma^{T^{l}} \in \Sigma^{T^{l}}$ for every $l=1, \ldots, \ell$ such that $\pi\left(\sigma^{T^{l}}, \tau^{N \backslash T^{l}}\right) \in B^{l}$ for all $\tau^{N \backslash T^{l}} \in \Sigma^{N \backslash T^{l}}$. Write $T:=$ 
$\bigcup_{l=1}^{\ell} T^{l}$, then $\pi\left(\sigma^{T}, \tau^{N \backslash T}\right) \in D$ for all $\tau^{N \backslash T} \in \Sigma^{N \backslash T}$. Since $D \in E^{H}(T)=$ $E^{\Gamma}(T)$ is minimal, there is $\sigma^{N \backslash T} \in \Sigma^{N \backslash T}$ with $\pi\left(\sigma^{N}\right)=x$. It is sufficient to show that $\sigma^{N} \in \operatorname{NE}\left(\Gamma, R^{N}\right)$. First, for every $l \in\{1, \ldots, \ell\}, i \in T^{l}$, and $\tau^{i} \in \Sigma^{i}$, we have $\pi\left(\sigma^{N \backslash\{i\}}, \tau^{i}\right) \in D^{l}$, hence $\pi\left(\sigma^{N}\right) R^{i} \pi\left(\sigma^{N \backslash\{i\}}, \tau^{i}\right)$ since $x R^{i} D^{l}$. Second, for every $i \in T$ and $\tau^{i} \in \Sigma^{i}$, we have $\pi\left(\sigma^{N \backslash\{i\}}, \tau^{i}\right) \in D$, hence $\pi\left(\sigma^{N}\right) R^{i} \pi\left(\sigma^{N \backslash\{i\}}, \tau^{i}\right)$ since $x R^{i} D$. So $\sigma^{N}$ is a Nash equilibrium of $\left(\Gamma, R^{N}\right)$.

\section{Examples and independence}

In this section, we present examples of social choice correspondences to which the results of Sections 3 and 4 apply. Furthermore, we show that the conditions in these results are independent.

\subsection{Scoring rules}

Let $W(A)$ denote the set of all weak orders of $A$, i.e., the set of all complete and transitive binary relations. All preceding results extend to preference profiles from $W(A)^{N}$ instead of $L(A)^{N}$ if we interpret all conditions as applying to the asymmetric parts of preferences in $W(A)$. Allowing indifferencesas in elements of $W(A)$-is convenient for the now following definition of scoring rules.

Let $|A|=m(\geq 2)$. A score vector is a vector $s=\left(s_{1}, \ldots, s_{m}\right)$ of nonnegative real numbers such that $s_{1} \leq \ldots \leq s_{m}$ and $s_{1}<s_{m}$. For a preference $R \in W(A)$ and an alternative $x \in A$, let $\ell(x, R):=|L(x, R)|$ and $\ell^{0}(x, R):=|\{y \in L(x, R) \mid x \notin L(y, R)\}|$. Then the score of $x$ at preference $R$ is defined by

$$
\operatorname{score}(x, R):=\frac{\sum_{k=\ell^{0}(x, R)+1}^{\ell(x, R)} s_{k}}{\ell(x, R)-\ell^{0}(x, R)} .
$$

So scores are assigned according to the vector $s$ with the understanding that equally preferred alternatives obtain the average of the associated scores. For $R^{N} \in W(A)^{N}$ and $x \in A$ we define

$$
\operatorname{score}\left(x, R^{N}\right)=\sum_{i \in N} \operatorname{score}\left(x, R^{i}\right) .
$$


For $k=1, \ldots, m$ define $\bar{s}^{k}:=\frac{1}{k}\left(s_{1}+\cdots+s_{k}\right)$. The social choice correspondence $H^{s}$ is defined by

$$
H^{s}\left(R^{N}\right):=\left\{x \in A \mid \operatorname{score}\left(x, R^{N}\right) \geq n \bar{s}^{m}\right\},
$$

hence it assigns to every preference profile $R^{N} \in W(A)^{N}$ all alternatives of average score or higher. Obviously, $H^{s}$ is well-defined: for every $R^{N} \in$ $W(A)^{N}, H^{s}\left(R^{N}\right) \neq \emptyset$.

Lemma 5.1 The SCC $H^{s}$ is monotone and surjective. It is unanimous if and only if $s_{m-1}<\bar{s}^{m}$. If $n>m$, then $E^{H^{s}}(N \backslash\{i\})=P(A)$ for every $i \in N$.

Proof. Monotonicity of $H^{s}$ is obvious. For surjectivity, let $x \in A$ and consider the profile $R^{N}$ where every player puts $x$ strictly on top and is indifferent between all other alternatives. Then $H^{s}\left(R^{N}\right)=\{x\}$.

For unanimity, it is necessary and sufficient that in a profile with $x \in A$ on top and $y \in A$ strictly ranked second for every player, only $x$ is chosen. This is the case if, and only if, $n s_{m-1}<n \bar{s}^{m}$, hence $s_{m-1}<\bar{s}^{m}$.

Before proving the last statement, first observe that for all $B \in P(A)$ and $T \in P(N)$, we have

$$
B \in E^{H^{s}}(T) \Leftrightarrow(n-|T|) s_{m}+|T| \bar{s}^{m-|B|}<n \bar{s}^{m} .
$$

(This can be seen by considering a profile where the players in $T$ put $B$ on top and are indifferent between the alternatives not in $B$, and the players not in $T$ all put the same alternative from $A \backslash B$ on top: this alternative should not be chosen.) To prove the last statement in the lemma, assume $n>m$. We have to show that $N \backslash\{i\}$ is effective for every singleton, hence by (5) that

$$
s_{m}+(n-1) \bar{s}^{m-1}<n \bar{s}^{m} .
$$

By a few elementary computations (in which $n>m$ is used) it follows that this inequality is equivalent to the inequality $\bar{s}^{m-1}<s_{m}$, which is true by definition of $s$.

Note that Lemma 5.1 implies that $H^{s}$ satisfies (S1) if $n>m$.

The next lemma provides a condition under which the SCC $H^{s}$ satisfies condition (S2). This condition is satisfied, for instance, if the assignment of scores in $s$ is determined by a nondecreasing convex function on the interval $[1, m]$. 
Lemma 5.2 Let the score vector s satisfy

$$
\bar{s}^{m} \leq \frac{s_{m}+s_{1}}{2} .
$$

Then $H^{s}$ satisfies (S2).

Proof. Let $B \in P(A) \backslash\{A\}$ and $T \in P(N)$. Then $B \in E^{H^{s}}(T)$ implies by (5) that

$$
(n-|T|) s_{m}+|T| \bar{s}^{m-|B|}<n \bar{s}^{m}
$$

hence

$$
|T|>\frac{n s_{m}-n \bar{s}^{m}}{s_{m}-\bar{s}^{m-|B|}} \geq \frac{n s_{m}-n \bar{s}^{m}}{s_{m}-s_{1}} \geq \frac{n}{2},
$$

where the last inequality follows from (6).

Let $b \in B \in E^{H^{s}}(T)$ and $R^{N} \in W(A)^{N}$ such that $b$ is strictly preferred to $A \backslash\{b\}$ for all $i \in T$ and $b$ is strictly preferred to $B \backslash\{b\}$ for all $i \in N \backslash T$. In order to prove (S2) it is, in view of (7), sufficient to show that $b \in H^{s}\left(R^{N}\right)$ or, equivalently, $|T| s_{m}+(n-|T|) s_{|B|} \geq n \bar{s}^{m}$. Suppose this were not true, then $|T| s_{m}+(n-|T|) \bar{s}^{|B|}<n \bar{s}^{m}$. Hence, by (5), $A \backslash B \in E^{H^{s}}(N \backslash T)$. Since $B \in E^{H^{s}}(T)$, this contradicts supperadditivity of $E^{H^{s}}$.

By Proposition 4.3 and Lemmas 5.1 and 5.2 we obtain:

Corollary 5.3 Let $n>m$ and let the score vector s satisfy (6). Then $H^{s}$ is constitutionally implementable.

Thus, Corollary 5.3 presents a class of surjective but not necessarily unanimous SCCs to which the results of Section 4 apply.

If, in addition to the conditions in Corollary 5.3, we have $s_{m-1}<\bar{s}^{m}$, then $H^{s}$ is unanimous by Lemma 5.1. This lemma also implies that (U2) is satisfied. Moreover, (7) implies that (U1) holds. So also Proposition 3.4 applies in this case, and we have a class of SCCs to which the results of Section 3 apply.

Alternatively, we can consider the Pareto optimal subcorrespondence $H_{P O}^{s}$ defined by

$$
\begin{aligned}
H_{P O}^{s}\left(R^{N}\right):= & \left\{x \in A \mid \operatorname{score}\left(x, R^{N}\right) \geq n \bar{s}^{m}\right. \\
& \text { and } \left.x \text { is Pareto undominated in } R^{N}\right\},
\end{aligned}
$$


for every $R^{N} \in W(A)^{N}$. Then $H_{P O}^{s}$ is well-defined, unanimous and monotone. For $n>m$ it satisfies (U2), and under (6), (U1) holds as well, in view of $(7)$. These claims can be verified by the same arguments as above. So Proposition 3.4 applies:

Corollary 5.4 Let $n>m$ and let the score vector s satisfy (6). Then $H_{P O}^{s}$ is constitutionally implementable.

Thus, Corollary 5.4 presents a class of unanimous SCCs to which the results of Section 3 apply.

\subsection{Veto rules}

In this section attention will be restricted again to linear orderings. We will exhibit another class of surjective SCCs to which the results of Section 4 apply.

Let $v$ be an integer with $v \geq 1$. We assume that $(m-1) v \leq n-1<$ $n<m v$. Say that a coalition $S$ vetoes an alternative $x \in A$ at a profile $R^{N} \in L(A)^{N}$ if there is a $T \subseteq S$ with $A R^{i} x$ for all $i \in T$ and $|T| \geq v$. Define the correspondence $H^{v}$ by

$$
H^{v}\left(R^{N}\right):=\{x \in A \mid \text { there is no } S \in P(N) \text { that vetoes } x\},
$$

for every $R^{N} \in W(A)^{N}$. Then $H^{v}$ is a well-defined social choice correspondence: in particular, $H^{v}\left(R^{N}\right) \neq \emptyset$ for every $R^{N} \in W(A)^{N}$ since $n<m v$. Further, $H^{v}$ is monotone, and surjective since $(m-1) v<n$. If every player puts an alternative $a$ on top and $b$ next, then both $a$ and $b$ are chosen provided that $m \geq 3$ : so in that case, $H^{v}$ is not unanimous.

Observe that for all $B \in P(A)$ and $S \in P(N)$

$$
B \in E^{H^{v}}(S) \Leftrightarrow|S| \geq(m-|B|) v .
$$

In particular, as $n-1 \geq(m-1) v$, this implies $E^{H^{v}}(N \backslash\{i\})=P(A)$ for every $i \in N$, so $H^{v}$ satisfies (S1).

Lemma $5.5 H^{v}$ satisfies (S2). 
Proof. With notations as in the definition of (S2) we have to prove $x \in$ $H^{v}\left(R^{N}\right)$. We assume without loss of generality that $B^{l} \neq A$ for every $l=$ $1, \ldots, \ell$. We write $T:=\bigcup_{l=1}^{\ell} T^{l}$.

If $|D| \geq 2$, then not $A R^{i} x$ for all $i \in N$. So $x$ is never a bottom element, hence $x \in H^{v}\left(R^{N}\right)$.

Suppose $D=\{x\}$. Then $\left|B^{1}\right|+\cdots+\left|B^{\ell}\right| \leq 1+(\ell-1) m$, so

$$
\begin{aligned}
|T|=\sum_{l=1}^{\ell}\left|T^{l}\right| & \geq \sum_{l=1}^{\ell}\left(m-\left|B^{l}\right|\right) v \\
& =v\left(\ell m-\sum_{l=1}^{\ell}\left|B^{l}\right|\right) \\
& \geq v(\ell m-1-(\ell-1) m) \\
& =(m-1) v,
\end{aligned}
$$

where the first inequality follows from (8). Hence, $n-|T| \leq n-(m-1) v<v$, which implies that $N \backslash T$ cannot veto $x$.

Suppose that $\left|D^{l}\right|=1$ for some $l \in\{1, \ldots, \ell\}$. Then by (8), $\left|T \backslash T^{l}\right| \geq$ $(m-1) v$. Hence, $\left|T^{l}\right|=|T|-\left|T \backslash T^{l}\right| \leq n-(m-1) v<v$, but also $\left|T^{l}\right| \geq$ $\left(m-\left|B^{l}\right|\right) v$ by $(8)$. Hence $\left(m-\left|B^{l}\right|\right) v<v$, a contradiction since $\left|B^{l}\right|<m$. We conclude that $\left|D^{l}\right| \geq 2$ for every $l=1, \ldots, \ell$ and so $x$ is not a bottom element of $R^{i}$ for every $i \in T$.

Hence, $x$ is not vetoed by any coalition, so $x \in H^{v}\left(R^{N}\right)$.

So, by Proposition 4.3, we obtain another class of constitutionally implementable surjective SCCs.

Corollary 5.6 Let $v$ be an integer with $v \geq 1$ and $(m-1) v \leq n-1<n<$ $m v$. Then $H^{v}$ is constitutionally implementable.

With appropriate restrictions on $v$ this result can be extended to veto rules where other than bottom alternatives can be vetoed.

\section{$5.3 \quad$ Independence}

We first show that the conditions in Proposition 3.4 are independent.

For each $R^{N} \in L(A)^{N}$ define the relative majority rule $H_{R M}$ by

$$
H_{R M}\left(R^{N}\right):=\left\{x \in A||\left\{i \in N \mid x R^{i} A\right\}|\geq|\left\{i \in N \mid y R^{i} A\right\} \mid \text { for all } y \in A\right\} \text {. }
$$


Then $H_{R M}$ is unanimous and satisfies (U1) and (U2), but it is not monotone.

For $n=5, m=3$, and $s=(1,2,3)$, the scoring rule $H^{s}$ is monotone and satisfies (U1) and (U2), but it is not unanimous.

The SCC $H_{1}$, defined by

$$
H_{1}\left(R^{N}\right):=\left\{x \in A||\left\{i \in N \mid x R^{i} A\right\} \mid \geq 1\right\}
$$

for every $R^{N} \in L(A)^{N}$, is unanimous, monotone, and satisfies (U1), but not (U2). (Note that $E^{H_{1}}(S)=\{A\}$ for all $S \in P(N)$ with $S \neq N$.)

For $n=8, m=3(A=\{a, b, c\})$, and $s=(0,2,3)$ consider the rule $\tilde{H}$ defined by

$$
\tilde{H}\left(R^{N}\right):=\left\{x \in A \mid x \text { is Pareto undominated and } \operatorname{score}\left(x, R^{N}\right) \geq 14\right\}
$$

for every $R^{N} \in(A)^{N}$. Then, obviously, $\tilde{H}\left(R^{N}\right) \neq \emptyset$ and $\tilde{H}$ is monotone and unanimous. Consider a profile $R^{N}$ with $a R^{i} b R^{i} c$ for four different players $i$ and with $a R^{i} c R^{i} b$ for three different players $i$. Then $\{a\}=\tilde{H}\left(R^{N}\right)$; since $a$ was arbitrary, this proves (U2). Finally, consider a profile $R^{N}$ with $a R^{i} b R^{i} c$ for all $i=1, \ldots, 4$. Then $\operatorname{score}\left(c, R^{N}\right) \leq 12$, so $c \notin \tilde{H}\left(R^{N}\right)$ and $\{a, b\} \in$ $E^{\tilde{H}}(\{1,2,3,4\})$. Similarly, $\{a, b\} \in E^{\tilde{H}}(\{5,6,7,8\})$, so we have a violation of (U1).

In order to prove logical independence of the conditions (S1), (S2), and monotonicity in Proposition 4.3 (we cannot drop surjectivity otherwise the associated effectivity function is not well-defined) we may use the same examples as above.

The relative majority rule $H_{R M}$ is not monotone and satisfies (S1) and (S2) (observe that (S1) is always implied by (U2)). The SCC $H_{1}$ is monotone and satisfies (S2) but not (S1). Finally, the SCC $\tilde{H}$ is monotone and satisfies (S1) but not (S2): to see this, recall that $\{a, b\} \in E^{\tilde{H}}(\{1,2,3,4\})$ and $\{a, b\} \in$ $E^{\tilde{H}}(\{5,6,7,8\})$. Take $T^{1}=\{1,2,3,4\}$ and $T^{2}=\{5,6,7,8\}$, and $B^{1}=B^{2}=$ $\{a, b\}$ in the definition of (S2), and let $a$ be the point $x$. Consider the profile $R^{N}$ where each player ranks $c$ above $a$ and $a$ above $b$. Then $\tilde{H}\left(R^{N}\right)=\{c\}$, which is a violation of $(\mathrm{S} 2)$.

\section{Concluding remarks}

In this paper we have obtained a tight characterization of all unanimous social choice correspondences that are constitutionally implementable. Fur- 
ther, we have established a tight set of sufficient conditions for constitutional implementability of social choice correspondences that are not necessarily unanimous but only surjective. Necessity of in particular condition (S2) is still an open problem.

\section{References}

Abdou, J., and H. Keiding (1991): Effectivity Functions in Social Choice. Dordrecht, The Netherlands: Kluwer Academic Publishers.

Danilov, V. (1992): "Implementation via Nash Equilibria," Econometrica, $60,43-56$.

Gaertner, W., P.K. Pattanaik, and K. Suzumura (1992): "Individual Rights Revisited," Economica, 59, 161-177.

Gärdenfors, P. (1981): "Rights, Games, and Social Choice," Nô̂s, 15, 341356.

Hurwicz, L. (1972): "On Informationally Decentralized Systems, in: Radner, R., and C.B. McGuire (eds.), Decision and Organization, in Honor of J. Marschak. Amsterdam: North-Holland, 297-336.

Maskin, E. (1985): "The Theory of Implementation in Nash Equilibrium," in: Hurwicz, L., D. Schmeidler, and H. Sonnenschein (eds.), Social Goals and Organization: Essays in Memory of Elisha Pazner. Cambridge: Cambridge University Press, 173-204.

Moulin, H., and B. Peleg (1982): "Cores of Effectivity Functions and Implementation Theory," Journal of Mathematical Economics, 10, 115-145.

Peleg, B. (1984): Game Theoretic Analysis of Voting in Committees. Cambridge: Cambridge University Press.

Peleg, B. (1998): "Effectivity Functions, Game Forms, Games, and Rights," Social Choice and Welfare, 15, 67-80.

Peleg, B., and E. Winter (2002): "Constitutional Implementation," Review of Economic Design, 7, 187-204.

Yamamoto, T. (1992): "On Nash Implementation of Social Choice Correspondences," Games and Economic Behavior, 4, 484-492. 